\title{
Gonadal macrophage infiltration in congenital lipoid adrenal hyperplasia
}

\author{
Tomohiro Ishii ${ }^{1}$, Ryuji Fukuzawa², Takeshi Sato ${ }^{1,4}$, Koji Muroya ${ }^{4}$, Masanori Adachi ${ }^{4}$, \\ Kenji Ihara', Junko Igaki ${ }^{3}$, Yukihiro Hasegawa ${ }^{3}$, Seiji Sato ${ }^{6}$, Toshikatsu Mitsuii and \\ Tomonobu Hasegawa ${ }^{1}$
}

${ }^{1}$ Department of Pediatrics, Keio University School of Medicine, Tokyo, Japan, ${ }^{2}$ Department of Pathology and Laboratory Medicine, ${ }^{3}$ Department of Endocrinology and Metabolism, Tokyo Metropolitan Children's Medical Center, Tokyo, Japan, ${ }^{4}$ Department of Endocrinology and Metabolism, Kanagawa Children's Medical Center, Yokohama, Japan, ${ }^{5}$ Department of Pediatrics, Graduate School of Medical Sciences, Kyushu University, Fukuoka, Japan, and ${ }^{6}$ Department of Pediatrics, Saitama Municipal Hospital, Saitama, Japan

Correspondence
should be addressed
to T Ishii
Email
tishii@1992.jukuin.keio.ac.jp

\begin{abstract}
Objective: Congenital lipoid adrenal hyperplasia (lipoid $\mathrm{CAH}$ ) results in impairment of adrenal and gonadal steroidogenesis caused by STAR mutations. Our previous study revealed upregulation of genes associated with inflammatory or immune response and macrophage infiltration in the adrenal cortex of Star-knockout mice. This study aimed at investigating macrophage infiltration in the gonads from human patients with lipoid CAH. Design: This study includes seven patients with lipoid CAH who underwent gonadectomy: two XX women (age, 22 and 40 years) and five $X Y$ boys ( 1 year). Two women with ovarian cysts ( 32 and 40 years) and six boys with autopsy or tumor (1 year) were examined as controls. Immunohistochemical analysis of their gonads was performed to determine steroidogenic cells by NR5A1 or CYP17A1 and macrophages by IBA1 or CD68.

Results: An increased number of macrophages infiltrated into the ovaries of lipoid CAH and consisted of two subpopulations: one scattered within and around a layer of theca cells of maturing follicles and the other massively aggregated in the stroma. Abundant cytoplasmic lipid droplets were observed not only in the theca cells but also in the stromal macrophages. There was no significant difference in the number of macrophages in the testicular interstitium between lipoid CAH (95\% confidence interval $\left(95 \% \mathrm{Cl}: 19.3-47.7\right.$ per $\left.0.2 \mathrm{~mm}^{2}\right)$ and controls $(95 \% \mathrm{Cl}$ : 13.3-25.8 per $\left.0.2 \mathrm{~mm}^{2}\right)(P=0.10)$.
\end{abstract}

Conclusions: These results demonstrate that macrophages infiltrate the ovaries of lipoid $\mathrm{CAH}$, where the theca cells and the stromal macrophages have abundant cytoplasmic lipid droplets.

\section{Introduction}

Steroidogenic acute regulatory protein (STAR) regulates the translocation of cholesterol from the outer to the inner mitochondrial membrane, where it serves as a common precursor of adrenal or gonadal steroid hormones under the acute stimulation of trophic hormones $(1,2)$. The physiological significance of STAR in steroidogenesis was confirmed by the fact that patients with STAR mutations (congenital lipoid adrenal hyperplasia, lipoid CAH) $(3,4,5)$ and knockout mice lacking Star (Star ${ }^{-1-}$ mice) $(6,7,8)$ both had significant defects in the biosynthesis of steroid hormone and diffuse accumulation of lipid droplets in the steroidogenic cells of the adrenal glands and gonads.

By conducting a transcriptome analysis of Star ${ }^{-1}$ mice, we previously demonstrated that a STAR deficiency altered the gene expression profile of adrenocortical cells (9). The significant alteration included the upregulation
() 2016 European Society of Endocrinology Printed in Great Britain
Published by Bioscientifica Ltd. 
of genes associated with cholesterol efflux as well as a previously undescribed inflammatory or immune response. We also identified increased macrophage infiltration in the adrenal cortex of Star ${ }^{-1}$ mice and proposed a paracrine interaction between adrenocortical cells and macrophages in cases of STAR deficiency (9), although studies on other species or tissues are required to confirm whether these conditions are ubiquitous consequences of STAR deficiency.

We therefore examined macrophage infiltration in the gonads of patients with lipoid $\mathrm{CAH}$ by immunohistochemical analyses. The present study expands our understanding of the pathophysiology of steroidogenic organs of STAR deficiency.

\section{Subjects and methods}

\section{Patients}

The present study involved seven patients diagnosed with lipoid $\mathrm{CAH}$ who underwent gonadectomy (Table 1). All patients or their parents provided informed consent to histological analysis of the extracted gonads and genetic analysis of the STAR gene. The protocol of the genetic analysis was approved by the Ethics Committee of Keio University School of Medicine. The direct sequencing of the STAR gene was performed for six patients, confirming homozygous or compound heterozygous mutations. The parents of patient 7 did not consent to genetic analysis. This patient was diagnosed with primary adrenal insufficiency with fatty deposition in the hyperplastic adrenal glands and complete female external genitalia with a $46, \mathrm{XY}$ karyotype. All patients exhibited symptoms of adrenal insufficiency with elevation of plasma ACTH and renin concentrations, and hydrocortisone and fludrocortisone replacement therapy was initiated before 1 year of age. These data indicate that all patients involved in this study showed the classic phenotype of lipoid CAH.

All the patients underwent gonadectomy. In two patients with a 46,XX karyotype, the ovaries were removed for different reasons. Patient 1 exhibited recurrent abdominal pain due to bilateral ovarian torsion and underwent right and left ovariectomies at 19 and 22 years of age, respectively (10). She had menarche at 11 years of age and started estrogen and progestin replacement therapy at 19 years of age. Patient 2 suffered from endometrioid adenocarcinoma and underwent total hysterectomy and bilateral adnexectomy at 40 years of age. She had menarche at 13 years of age and started estrogen and progestin replacement therapy at 25 years of age. In five patients with a 46,XY karyotype $(47, \mathrm{XY},+21$ for Patient 6), the testes were removed before 2 years of age, because they were reared as girls with complete female external genitalia.

\section{Controls}

The testicular tissues of six boys at 1 year of age (with autopsy in two or with gonadectomy because of testicular tumor in four) and the ovarian tissues of 32- and 40-yearold women with ovarian cysts were examined. All controls or their parents provided informed consent to analyze these tissues.

Table 1 Clinical and pathological findings of seven patients with congenital lipoid adrenal hyperplasia.

\begin{tabular}{|c|c|c|c|c|c|c|c|}
\hline Case & 1 & 2 & 3 & 4 & 5 & 6 & 7 \\
\hline Karyotype & $46, X X$ & $46, X X$ & $46, X Y$ & $46, X Y$ & $46, X Y$ & $47, X Y,+21$ & $46, X Y$ \\
\hline STAR genotype & Q258*/Q258* & Q258*/E64fs & Q258*/Q258* & Q258*/Q258* & Q258*/R272P & Q258*/Q258* & NA \\
\hline $\begin{array}{l}\text { Age at gonadec- } \\
\text { tomy (years) }\end{array}$ & 22 & 40 & 1 & 1 & 1 & 1 & 1 \\
\hline $\begin{array}{l}\text { Lipid droplets in } \\
\text { the theca cells }\end{array}$ & + & + & NA & NA & NA & NA & NA \\
\hline $\begin{array}{l}\text { Lipid droplets in } \\
\text { the Leydig cells }\end{array}$ & NA & NA & - & + & + & + & + \\
\hline $\begin{array}{l}\text { Number of } \\
\text { macrophage in } \\
\text { gonads }\end{array}$ & Increased $^{a}$ & Increased $^{a}$ & $38.0 \pm 4.4^{b, c}$ & $6.0 \pm 1.3^{b, d}$ & $47.2 \pm 4.9^{b, c}$ & $33.8 \pm 4.4^{b}$ & $42.6 \pm 4.2^{b, c}$ \\
\hline $\begin{array}{l}\text { Reason for } \\
\text { gonadectomy }\end{array}$ & $\begin{array}{l}\text { Torsion of } \\
\text { ovarian cyst }\end{array}$ & $\begin{array}{l}\text { Endometrioid } \\
\text { adenocarcinoma }\end{array}$ & Reared female & Reared female & Reared female & Reared female & Reared female \\
\hline
\end{tabular}




\section{Histological analyses}

Histological characteristics were examined with sections from formalin-fixed or frozen gonadal tissue specimens. The general structure and lipid deposition of the gonadal tissues were assessed by hematoxylin-eosin (HE) and oil red $\mathrm{O}$ stainings, respectively. Immunohistochemistry for steroidogenic factor 1 (NR5A1) was used as a marker of steroidogenic cells, and that for ionized calciumbinding adaptor molecule 1 (IBA1) or CD68 was employed as a marker of macrophages. To distinguish between different types of steroidogenic cell in the ovary, 17 $\alpha$-hydroxylase (CYP17A1) was used as a marker of theca cells. The following primary antibodies were used: mouse monoclonal antibody against NR5A1 (Perseus Proteomics, Tokyo, Japan) at 1 in 100 dilution, rabbit anti-IBA1 polyclonal antibodies (Wako Pure Chemical Industries) at 1 in 4000 dilution, goat anti-CYP17A1 polyclonal antibodies (LifeSpan BioSciences, Inc, Seattle, WA, USA) at 1 in 100 dilution, and mouse anti-CD68 monoclonal antibody (Nichirei, Tokyo, Japan; diluted). The anti-IBA1 polyclonal antibodies, which worked only in formalin-fixed tissues, detected macrophages more specifically than anti-CD68 monoclonal antibody (11). For immunoenzyme detection in double staining, NR5A1 and IBA1 were visualized by horseradish peroxidase using diaminobenzidine and alkaline phosphatase using Permanent Red as chromogen, respectively (EnVision G2 Doublestein System Rabbit/Mouse, Dako). For immunofluorescence detection in double staining, the following secondary antibodies were used: anti-mouse IgG $\left.(\mathrm{H}+), \mathrm{F}_{(\mathrm{ab}}\right)_{2}$ fragment (AlexaFluor 488 Conjugate) (Cell Signaling), anti-rabbit IgG $(\mathrm{H}+), \mathrm{F}\left(\mathrm{ab}^{\prime}\right)_{2}$ fragment (AlexaFluor 555 Conjugate) (Cell Signaling), and anti-goat IgG $(\mathrm{H}+), \mathrm{F}\left(\mathrm{ab}^{\prime}\right)_{2}$ fragment (AlexaFluor 350 Conjugate) (Cell Signaling). For immunoenzyme detection in single staining, CYP17A1 or CD68 was visualized by horseradish peroxidase using diaminobenzidine.

\section{Results}

\section{Ovary}

Histological analysis revealed similar features in the ovaries of two adult patients with lipoid CAH (patients 1 and 2). These features included the maturing follicles with the hyperplastic theca cells (Fig. 1). The granulosa cells, which should be present in the inner layer of the theca cells, were occasionally not clearly identified (data not shown).

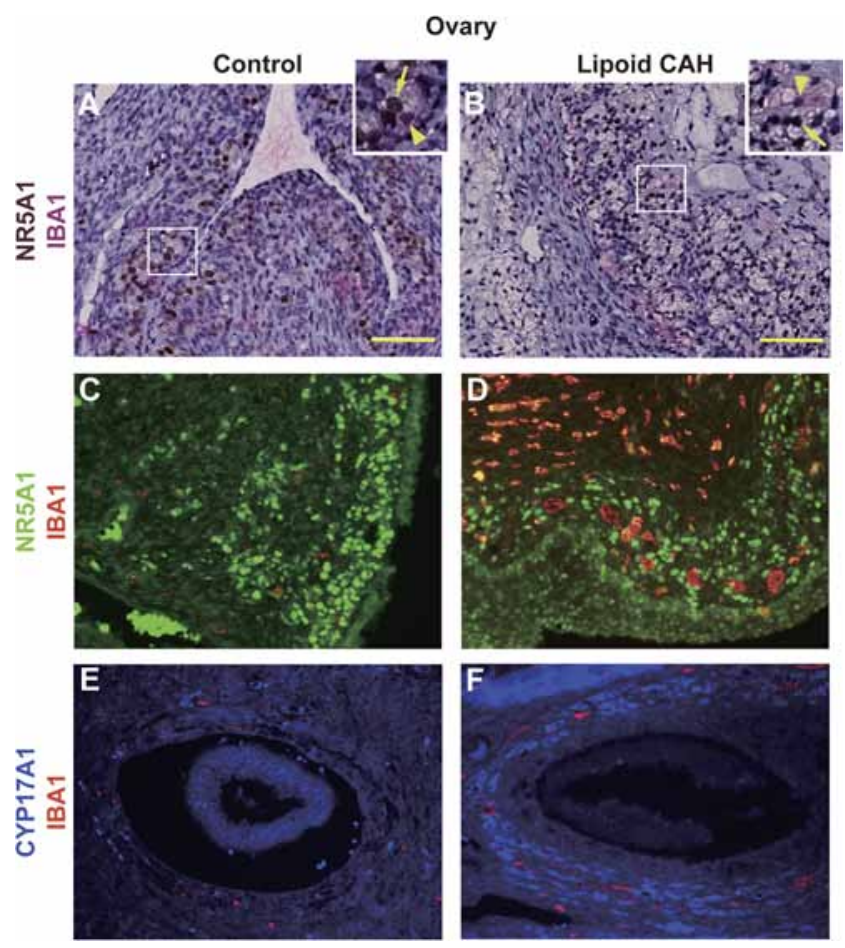

\section{Figure 1}

Immunohistochemical localization of steroidogenic cells and macrophages in the formalin-fixed adult ovaries.

Steroidogenic factor 1 (NR5A1) is a marker for granulosa and theca cells, 17 $\alpha$-hydroxylase (CYP17A1) for theca cells, and ionized calcium-binding adaptor molecule 1 (IBA1) for resident macrophages. $A, C$, and $E$, ovary of a control; $B, D$, and $F$, ovary of a patient with congenital lipoid adrenal hyperplasia. A and B, magenta and brown colors for immunoreactivities against IBA1 and NR5A1, respectively; $C$ and $D$, red and green fluorescences for IBA1 and NR5A1, respectively; E and F, red and blue fluorescences for IBA1 and CYP17A1, respectively. An inset shows the magnified view of each white rectangle. Yellow arrows and arrowheads indicate steroidogenic cells and macrophages, respectively. Yellow bars indicate $73 \mu \mathrm{m}$. Original magnification was $200 \times$ for $A$ and $B$, and $400 \times$ for $C-F$.

The immunohistochemical analyses clarified localization of macrophages in the ovaries (Fig. 1). These macrophages infiltrated sparsely in the control ovaries; compared with the control, the number of macrophages was markedly increased in the ovaries of those with lipoid CAH. These macrophages were located in patchy distribution, hardly allowing quantitative assessment between control and patients, and divided into two subpopulations. One group of macrophages was detected in the layer of follicular epithelia, closely to the theca 


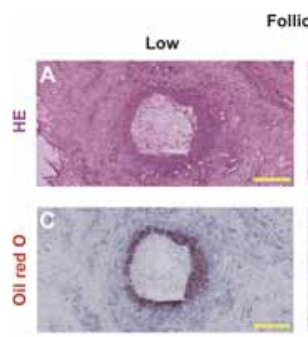

Follicle High
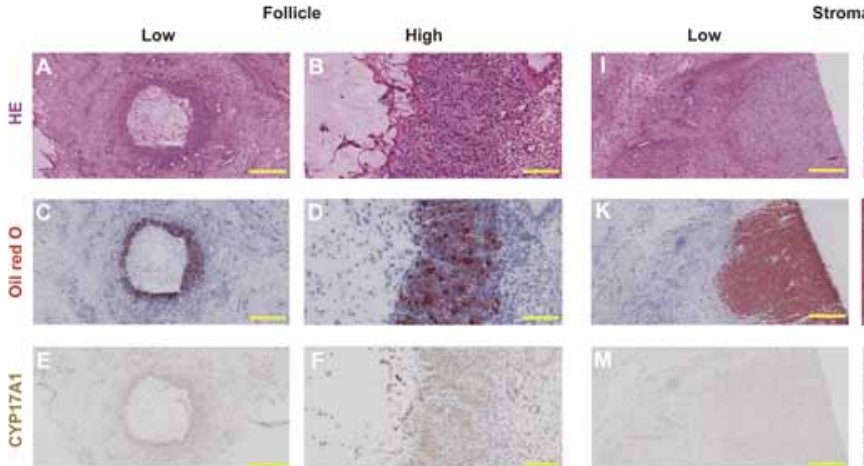

Stroma

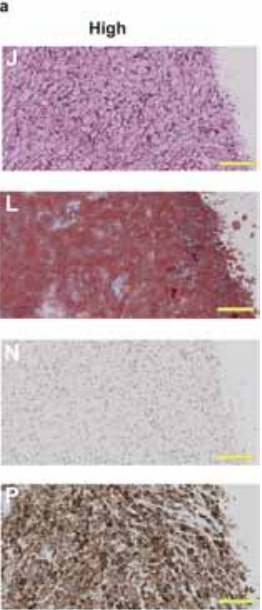

\section{Figure 2}

Immunohistochemical localization of steroidogenic cells and macrophages and distribution of lipid droplets in the fresh-frozen adult ovary of congenital lipoid adrenal hyperplasia (lipoid CAH). Four consecutive sections were stained differently. Oil red $\mathrm{O}$ staining is indicative of lipid droplets as fresh red. 17 $\alpha$-hydroxylase (CYP17A1) is a marker for theca cells, and CD68 for resident macrophages. A-H, ovarian follicle; I-P, ovarian stroma. A, B, I, and H, hematoxylin-eosin staining; C, D, K, and L, oil red O staining; E, F, M, and N, light brown colors for immunoreactivities against CYP17A1; G, H, O, and P, dark brown colors for immunoreactivities against CD68. Yellow bars indicate $500 \mu \mathrm{m}$ for $\mathrm{A}, \mathrm{C}, \mathrm{E}, \mathrm{G}, \mathrm{I}, \mathrm{K}, \mathrm{M}$, and $\mathrm{O}$ (original magnification was 40x) and $100 \mu \mathrm{m}$ for $\mathrm{B}, \mathrm{D}, \mathrm{F}, \mathrm{H}, \mathrm{J}, \mathrm{L}, \mathrm{N}$, and $\mathrm{P}$ (original magnification was $200 \times$ ).

cells that expressed NR5A1 and CYP17A1 (follicular macrophages); the other group massively aggregated in stroma and was probably associated with atretic follicles or corpora albicans, apart from other steroidogenic cells that expressed NR5A1 or CYP17A1 (stromal macrophages).

The oil red $\mathrm{O}$ staining and CD68 immunostaining using consecutive sections revealed the positional overlap between the lipid droplets and the macrophages (Fig. 2). The stromal macrophages with cytoplasmic lipid droplets were even predominantly observed compared with the theca cells with cytoplasmic lipid droplets. No steroidogenic cells were observed among or around the stromal macrophages. The lipid-laden cells in the follicular layer partly appeared to originate from the theca cells because they were immuno-positive for CYP17A1. We could not determine whether or not the follicular macrophages also had cytoplasmic lipid droplets, because these macrophages and the lipid-laden theca cells resided too closely to distinguish them.

\section{Testis}

The histological analysis of HE sections revealed fewer seminiferous tubules and fewer germ cells in patients with lipoid CAH than in controls as reported previously (12). In the XY patients (except for Patient 3), the Leydig cells in the interstitium had a foamy appearance. Any of the $\mathrm{XY}$ patients did not have Leydig cells hyperplasia that was reported in only a few patients with lipoid CAH (12).

The immunohistochemical analyses identified resident macrophages within the testicular interstitium of both controls and patients with lipoid CAH (Fig. 3). These macrophages spread through the interstitium, allowing quantitative assessment in a specific microscope field $\left(0.2 \mathrm{~mm}^{2}\right)$. There was no significant difference in the mean number of macrophages between the controls (95\% confidence interval, $13.3-25.8$ per $0.2 \mathrm{~mm}^{2}$ ) and patients (19.3-47.7) ( $P=0.10$ by Mann-Whitney test). Three out of five patients, however, had significantly more macrophages than the controls (Table 1).

\section{Discussion}

The present study revealed that the ovary of adult patients with lipoid CAH is characterized by prominent infiltration of macrophages with cytoplasmic limpid accumulation, although the macrophage infiltration did not differ significantly in the testis of infants between controls and patients. To our knowledge, this observation regarding ovarian macrophage in lipoid $\mathrm{CAH}$ has not been studied, partly because the ovarian specimens are difficult to obtain. These results 


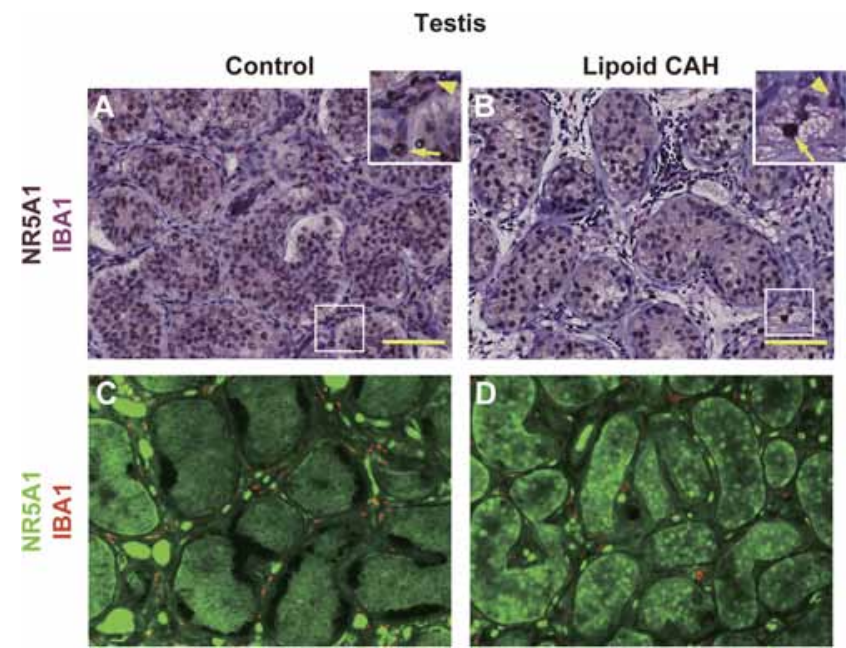

Figure 3

Immunohistochemical localization of steroidogenic cells and macrophages in the formalin-fixed infantile testes.

Steroidogenic factor 1 (NR5A1) is a marker for Sertoli and Leydig cells and ionized calcium-binding adaptor molecule 1 (IBA1) for resident macrophages. A and C, testis of a control; $B$ and $D$, testis of an infant with congenital lipoid adrenal hyperplasia. $A$ and $B$, magenta and brown colors for immunoreactivities against IBA1 and NR5A1, respectively; $C$ and $D$, red and green fluorescences for IBA1 and NR5A1, respectively. An inset shows the magnified view of each white rectangle. Yellow arrows and arrowheads indicate steroidogenic cells and macrophages, respectively. Yellow bars indicate $73 \mu \mathrm{m}$. Original magnification was $200 \times$ for $A$ and $B$, and $400 \times$ for $C$ and $D$.

are consistent with our previous observation that macrophages infiltrated the adrenal cortex of fetal Star ${ }^{-1-}$ mice (9), thereby suggesting that this phenomenon is common for STAR deficiency among different species and different steroidogenic tissues.

The macrophages in the ovaries could play a specific role in the pathophysiology of lipoid CAH. The excessive follicular atresia results in premature ovarian failure, which is one of the features described for $46, \mathrm{XX}$ adolescents or adults with lipoid CAH (3). The underlying mechanism of the atresia is explained by the two-hit model in which accumulated cholesterol esters abrogate the residual capacity of the STAR-independent steroidogenesis initially preserved in STAR-deficient steroidogenic cells (3). The follicular macrophages adjacent to the lipid-laden theca cells may modulate the adaptive response to excessive cholesterol accumulation. The aggregation of the lipidladen stromal macrophages could be the consequence of depletion and replacement for atretic follicle or corpus luteum in which cholesterol esters were accumulated abundantly. We described previously that the adrenal steroidogenic cells of Star $^{-1}$ mice had significantly increased the expression of genes involved in inflammatory or immune response (9), some of which have a pivotal role in the chronic inflammation in the adipose tissue of obese individuals (13). Thus, it is plausible to hypothesize the possible link between cholesterol accumulation and macrophage infiltration in the ovaries of lipoid CAH.

Several lines of evidence suggest that the paracrine interactions between macrophages and Leydig cells are physiologically relevant (14). In this study, three XY infants with lipoid $\mathrm{CAH}$ showed significant increase in macrophage infiltration, the degree of which was less obvious in the infant testes compared with that in the adult ovaries. This is partly explained by the notion that hyperplasia of Leydig cells in infants is less manifested than that of the theca cells in adult patients $(10,12)$. Intriguingly, testicular macrophages have the potential to provide 25-hydroxycholesterol as a direct substrate for P450 side-chain cleavage enzyme in an alternate pathway for steroidogenesis, which bypasses the STAR-dependent delivery of cholesterol into the inner mitochondrial membrane $(15,16)$. Thus, potential steroidogenic cooperation may also occur between the macrophages and the ovarian theca cells that, similar to the testicular Leydig cells, make androgens as well.

In summary, we have further demonstrated a potentially pivotal role of the inflammatory or immune response in pathological conditions of steroidogenic tissues. The ovaries and adrenal glands in patients with STAR deficiency may share some common features of homeotic inflammation with white adipose tissue in patients with obesity and with the vascular walls in those with atherosclerosis (17). This speculation needs to be proved by different strategies including in vitro experiment, and further questions still remain unsolved including where the macrophages come from and what is the role of macrophages in steroidogenic tissues of lipoid $\mathrm{CAH}$.

Declaration of interest

The authors declare that there is no conflict of interest that could be perceived as prejudicing the impartiality of the research reported.

\section{Funding}

This work was supported by Grant-in-Aid for Scientific Research (C) (Grant Numbers 23591516 and 16K09996) from the Japan Society for the 
Promotion of Science, a Health Science Research Grant for Research on Applying Health Technology (Jitsuyoka (Nanbyo)-Ippan-014) from the Ministry of Health, Labour and Welfare, Japan, and the Japan Foundation for Pediatric Research.

\section{Author contribution statement}

TI contributed to collection of the samples, analysis and interpretation of the data, and preparation of the manuscript. $R$ F contributed to histological analyses of the samples and interpretation of the data. T S, K $\mathrm{M}, \mathrm{M} \mathrm{A}, \mathrm{K} \mathrm{I}, \mathrm{JI}, \mathrm{Y} \mathrm{H}, \mathrm{S} \mathrm{S}$, and T M contributed to collection of the samples. $\mathrm{T} \mathrm{H}$ contributed to analysis and interpretation of the data and review of the manuscript.

\section{Acknowledgments}

The authors thank Drs Satoshi Narumi (Department of Pediatrics, Keio University School of Medicine, Tokyo, Japan) and Goro Sasaki (Department of Pediatrics, Tokyo Dental University, Ichikawa, Japan) for helpful discussion.

\section{References}

1 Stocco DM. StAR protein and the regulation of steroid hormone biosynthesis. Annual Review of Physiology 200163 193-213. (doi:10.1146/annurev.physiol.63.1.193)

2 Jefcoate C. High-flux mitochondrial cholesterol trafficking, a specialized function of the adrenal cortex. Journal of Clinical Investigation 2002110 881-890. (doi:10.1172/JCI0216771)

3 Bose HS, Sugawara T, Strauss JF \& Miller WL. The pathophysiology and genetics of congenital lipoid adrenal hyperplasia. New England Journal of Medicine 1996335 1870-1878. (doi:10.1056/ NEJM199612193352503)

4 Bose HS, Pescovitz OH \& Miller WL. Feminization in a 46, XX female patient with congenital lipoid adrenal hyperplasia due to a homozygous Frameshift Mutation in the steroidogenic acute regulatory protein. Journal of Clinical Endocrinology \& Metabolism 1997 82 1511-1515. (doi:10.1210/jcem.82.5.3962)

5 Fujieda K, Tajima T, Nakae J, Sageshima S, Tachibana K, Suwa S, Sugawara T \& Strauss JF. Spontaneous puberty in $46, \mathrm{XX}$ subjects with congenital lipoid adrenal hyperplasia. Ovarian steroidogenesis is spared to some extent despite inactivating mutations in the steroidogenic acute regulatory protein (StAR) gene. Journal of Clinical Investigation 199799 1265-1271. (doi:10.1172/JCI119284)

6 Hasegawa T, Zhao L, Caron KM, Majdic G, Suzuki T, Shizawa S, Sasano H \& Parker KL. Developmental roles of the steroidogenic acute regulatory protein (StAR) as revealed by StAR knockout mice. Molecular Endocrinology 200014 1462-1471. (doi:10.1210/ me.14.9.1462)

7 Ishii T, Hasegawa T, Pai C-I, Yvgi-Ohana N, Timberg R, Zhao L, Majdic G, Chung B-C, Orly J \& Parker KL. The roles of circulating high-density lipoproteins and trophic hormones in the phenotype of knockout mice lacking the steroidogenic acute regulatory protein Molecular Endocrinology 200216 2297-2309. (doi:10.1210/me.20010320)

8 Sasaki G, Ishii T, Jeyasuria P, Jo Y, Bahat A, Orly J, Hasegawa T \& Parker KL. Complex role of the mitochondrial targeting signal in the function of steroidogenic acute regulatory protein revealed by bacterial artificial chromosome transgenesis in vivo. Molecular Endocrinology 200822 951-964. (doi:10.1210/me.2007-0493)

9 Ishii T, Mitsui T, Suzuki S, Matsuzaki Y \& Hasegawa T. A genome-wide expression profile of adrenocortical cells in knockout mice lacking steroidogenic acute regulatory protein. Endocrinology $2012 \mathbf{1 5 3}$ 2714-2723. (doi:10.1210/en.2011-1627)

10 Kaku U, Kameyama K, Izawa M, Yamada M, Miyamoto J, Suzuki T, Sasano H \& Hasegawa Y. Ovarian histological findings in an adult patient with the steroidogenic acute regulatory protein (StAR) deficiency reveal the impairment of steroidogenesis by lipoid deposition. Endocrine Journal 200855 1043. (doi:10.1507/endocrj.K08E-102)

11 Guillemin GJ \& Brew BJ. Microglia, macrophages, perivascular macrophages, and pericytes: a review of function and identification. Journal of Leukocyte Biology 200475 388-397. (doi:10.1189/ jlb.0303114)

12 Aya M, Ogata T, Sakaguchi A, Sato S \& Matsuo N. Testicular histopathology in congenital lipoid adrenal hyperplasia: a light and electron microscopic study. Hormone Research 199747 121-125. (doi:10.1159/000185446)

13 Suganami T \& Ogawa Y. Adipose tissue macrophages: their role in adipose tissue remodeling. Journal of Leukocyte Biology 201088 33-39. (doi:10.1189/jlb.0210072)

14 Yee JB \& Hutson JC. Effects of testicular macrophage-conditioned medium on Leydig cells in culture. Endocrinology 1985116 2682-2684. (doi:10.1210/endo-116-6-2682)

15 Lukyanenko YO, Chen JJ \& Hutson JC. Production of 25-hydroxycholesterol by testicular macrophages and its effects on Leydig cells. Biology of Reproduction 200164 790-796. (doi:10.1095/ biolreprod64.3.790)

16 King SR, Matassa AA, White EK, Walsh LP, Jo Y, Rao RM, Stocco DM $\&$ Reyland ME. Oxysterols regulate expression of the steroidogenic acute regulatory protein. Journal of Molecular Endocrinology 200432 507-517. (doi:10.1677/jme.0.0320507)

17 Rocha VZ \& Libby P., Obesity inflammation, and atherosclerosis. Nature Reviews Cardiology 20096 399-409. (doi:10.1038/ nrcardio.2009.55)

Received 3 March 2016

Revised version received 11 May 2016

Accepted 17 May 2016 